CUBO A Mathematical Journal

Vol.12, № 03, (49-69). October 2010

\title{
On The Group of Strong Symplectic Homeomorphisms
}

\author{
AUgustin BANYAGa \\ Department of Mathematics, The Pennsylvania State University, \\ University Park, PA 16802 \\ email: banyaga@math.psu.edu
}

\begin{abstract}
We generalize the "hamiltonian topology" on hamiltonian isotopies to an intrinsic "symplectic topology" on the space of symplectic isotopies. We use it to define the group SSympeo $(M, \omega)$ of strong symplectic homeomorphisms, which generalizes the group $\operatorname{Hameo}(M, \omega)$ of hamiltonian homeomorphisms introduced by $\mathrm{Oh}$ and Müller. The group $\operatorname{SSympeo}(M, \omega)$ is arcwise connected, is contained in the identity component of $S y m p e o(M, \omega)$; it contains $\operatorname{Hameo}(M, \omega)$ as a normal subgroup and coincides with it when $M$ is simply connected. Finally its commutator subgroup $[S \operatorname{Sympeo}(M, \omega), \operatorname{SSympeo}(M, \omega)]$ is contained in $\operatorname{Hameo}(M, \omega)$.
\end{abstract}

\section{RESUMEN}

Generalizamos la "topología hamiltoniano" sobre isotopias hamiltonianas para una "topología simpléctica" intrinseca en el espacio de isotopias simplécticas. Nosotros usamos esto para definir el grupo $S S y m p e o(M, \omega)$ de homeomorfismos simplécticos fuertes, el qual generaliza el grupo $\mathrm{Hameo}(M, \omega)$ de homeomorfismos hamiltonianos introducido por $\mathrm{Oh}$ y Müller. El grupo $S \operatorname{Sympeo}(M, \omega)$ es conexo por arcos, es contenido en la componente identidad de $S y m p e o(H, \omega)$; este contiene $\operatorname{Hameo}(M, \omega)$ como un subgrupo normal y coincide con este cuando $M$ es simplemente conexa. Finalmente su subgrupo conmutador $[\operatorname{SSympeo}(M, \omega), \operatorname{SSympeo}(M, \omega)]$ es contenido en $\operatorname{Hameo}(M, \omega)$. 
Key words and phrases: Hamiltonian homeomorphisms, hamiltonian topology, symplectic topology, stromg symplectic homeomorphisms, $C^{0}$ symplectic topology.

Math. Subj. Class.: MSC2000:53D05; 53D35.

\section{Introduction}

No natural metric on the group $\operatorname{Symp}(M, \omega)$ of symplectic diffeomorphisms of a symplectic manifold $(M, \omega)$ is known. In this paper we construct a "Hofer-like" metric, depending on several ingredients. However, we prove that all these metrics are equivalent and hence define a natural metric topology on $\operatorname{Sym} p(M, \omega)$ ( theorem 1'). We use this natural topology on $\operatorname{Symp}(M, \omega)$ to define a new group of symplectic homeomorphisms, herein called the group of strong symplectic homeomorphisms (Theorem 2). This group may carry a Calabi invariant.

The Eliashberg-Gromov symplectic rigidity theorem says that the group $\operatorname{Symp}(M, \omega)$ of symplectomorphisms of a closed symplectic manifold $(M, \omega)$ is $C^{0}$ closed in the group $\operatorname{Diff}^{\infty}(M)$ of $C^{\infty}$ diffeomorphisms of $M$ [7],[9]. This means that the "symplectic" nature of a sequence of symplectomorphisms survives topological limits. Also Lalonde-McDuff-Polterovich have shown in [11] that for a symplectomorphism, being "hamiltonian" is topological in nature. These phenomenons attest that there is a $C^{0}$ symplectic topology underlying the symplectic geometry of a closed symplectic manifold $(M, \omega)$.

According to Oh-Müller ([13]), the automorphism group of the $C^{0}$ symplectic topology is the closure of the group $\operatorname{Symp}(M, \omega)$ in the group Homeo(M) of homeomorphisms of $M$ endowed with the $C^{0}$ topology. That group, denoted $\operatorname{Sympeo}(M, \omega)$ has been called the group of symplectic homeomorphisms:

$$
\operatorname{Sympeo}(M, \omega)=: \overline{\operatorname{Symp}(M, \omega)} .
$$

The $C^{0}$ topology on $H o m e o(M)$ coincides with the metric topology coming from the metric

$$
\bar{d}(g, h)=\max \left(\sup _{x \in M} d_{0}(g(x), h(x)), \sup _{x \in M} d_{0}\left(g^{-1}(x), h^{-1}(x)\right)\right.
$$

where $d_{0}$ is a distance on $M$ induced by some riemannian metric [3].

On the space $P H o m e o(M)$ of continuous paths $\gamma:[0,1] \rightarrow H o m e o(M)$, one has the distance

$$
\bar{d}(\gamma, \mu)=\sup _{t \in[0,1]} \bar{d}(\gamma(t), \mu(t)) .
$$

Consider the space $\operatorname{PHam}(M)$ of all isotopies $\Phi_{H}=\left[t \mapsto \Phi_{H}^{t}\right]$ where $\Phi_{H}^{t}$ is the family of hamiltonian diffeomorphisms obtained by integration of the family of vector fields $X_{H}$ for a 
smooth family $H(x, t)$ of real functions on $M$, i.e.

$$
\frac{d}{d t} \Phi_{H}^{t}(x)=X_{H}\left(\Phi_{H}^{t}(x)\right)
$$

and $\Phi_{H}^{0}=i d$.

Recall that $X_{H}$ is uniquely defined by the equation

$$
i\left(X_{H}\right) \omega=d H
$$

where $i($.$) is the interior product.$

The set of time one maps of all hamiltonian isotopies $\left\{\Phi_{H}^{t}\right\}$ form a group, denoted $\operatorname{Ham}(M, \omega)$ and called the group of hamiltonian diffeomorphisms.

Definition: The hamiltonian topology [13] on $\operatorname{PHam}(M)$ is the metric topology defined by the distance

$$
d_{\text {ham }}\left(\Phi_{H}, \Phi_{H^{\prime}}\right)=\left\|H-H^{\prime}\right\|+\bar{d}\left(\Phi_{H}, \Phi_{H^{\prime}}\right)
$$

where

$$
\left\|H-H^{\prime}\right\|=\int_{0}^{1} o s c\left(H-H^{\prime}\right) d t .
$$

and the oscillation of a function $u$ is

$$
o s c(u)=\max _{x \in M} u(x)-\min _{x \in M} u(x) .
$$

Let $\operatorname{Hameo}(M, \omega)$ denote the space of all homeomorphisms $h$ such that there exists a continuous path $\lambda \in P H o m e o(M)$ such that $\lambda(0)=i d, \lambda(1)=h$ and there exists a Cauchy sequence (for the $d_{\text {ham }}$ distance) of hamiltonian isotopies $\Phi_{H^{n}}$, which $C^{0}$ converges to $\lambda$ ( in the $\bar{d}$ metric).

The following is the first important theorem in the $C^{0}$ symplectic topology [13]:

Theorem (Oh-Müller): The set Hameo $(M, \omega)$ is a topological group. It is a normal subgroup of the identity component Sympeo $(M, \omega)$ in $\operatorname{Sympeo}(M, \omega)$. If $H^{1}(M, \mathbb{R}) \neq 0$, then $\operatorname{Hameo}_{(M, \omega)}$ is strictly contained in Sympeo $0(M, \omega)$.

Remark: It is still unknown in general if the inclusion

$$
\operatorname{Hameo}(M, \omega) \subset \operatorname{Sympeo}_{0}(M, \omega)
$$

is strict.

The group $\operatorname{Hameo}(M, \omega)$ is the topological analogue of the group $\operatorname{Ham}(M, \omega)$ of hamiltonian diffeomorphisms. 
The goal of this paper is to construct a subgroup of Sympeo $(M, \omega)$, denoted SSympeo $(M, \omega)$ and nicknamed the group of strong symplectic homeomorphisms, containing $\operatorname{Hameo}(M, \omega)$, that is:

$$
\operatorname{Hameo}(M, \omega) \subset \operatorname{SSympeo}(M, \omega) \subset \operatorname{Sympeo}_{0}(M, \omega) .
$$

Like Hameo $(M, \omega)$, the group $S S y m p e o(M, \omega)$ is defined using a blend of the $C^{0}$ topology and the Hofer topology on the space $I s o(M, \omega)$ of symplectic isotopies of $(M, \omega)$.

We believe that $\operatorname{SSympeo}(M, \omega)$ is "more right" than the group $\operatorname{Sympeo}(M, \omega)$ for the $C^{0}$ symplectic topology. In particular the flux homomorphism seems to exist on $\operatorname{SSympeo}(M, \omega)$. This will be the object of a future paper.

The results of this paper have been announced in [1].

The $C^{0}$ counter part of the $C^{\infty}$ contact topology is been worked out in [5], [6].

\section{The Symplectic Topology on $I s o(M, \omega)$}

Let $I s o(M, \omega)$ denote the space of symplectic isotopies of a closed symplectic manifold $(M, \omega)$. Recall that a symplectic isotopy is a smooth map $\Phi: M \times[0,1] \rightarrow M$ such that for all $t \in[0,1]$, $\phi_{t}: M \rightarrow M, \quad x \mapsto \Phi(x, t)$ is a symplectic diffeomorphism and $\phi_{0}=i d$.

The "Lie algebra" of $\operatorname{Symp}(M, \omega)$ is the space $\operatorname{sym} p(M, \omega)$ of symplectic vector fields, i.e the set of vector fields $X$ such that $i_{X} \omega$ is a closed form.

Let $\phi_{t}$ be a symplectic isotopy, then

$$
\dot{\phi}_{t}(x)=\frac{d \phi_{t}}{d t}\left(\phi_{t}^{-1}(x)\right)
$$

is a smooth family of symplectic vector fields.

By the theorem of existence and uniqueness of solutions of ODE's,

$$
\Phi \in I s o(M, \omega) \mapsto \dot{\phi}_{t}
$$

is a 1-1 correspondence between $\operatorname{Iso}(M, \omega)$ and the space $C^{\infty}([0,1] \operatorname{sym} p(M, \omega))$ of smooth families of symplectic vector fields. Hence any distance on $C^{\infty}([0,1] \operatorname{sym} p(M, \omega))$ gives rise to a distance on $I s o(M, \omega)$.

An intrinsic topology on the space of symplectic vector fields.

We define a norm $\|$.$\| on \operatorname{sym} p(M, \omega)$ as follows: first we fix a riemannian metric $g$ (which may be the one we used to define $d_{0}$ above, or any other riemannian metric), and a basis $\mathscr{B}=$ $\left\{h_{1}, \ldots, h_{k}\right\}$ of harmonic 1-forms. For Hodge theory, we refer to [14]. 
Recall that the space $\operatorname{harm}^{1}(M, g)$ of harmonic 1-forms is a finite dimensional vector space over $\mathbb{R}$ and its dimension is the first Betti number of $M$.

On $\operatorname{harm}^{1}(M, g)$, we put the following "Euclidean" norm:

for $\mathscr{H} \in \operatorname{harm}^{1}(M, g), \mathscr{H}=\sum \lambda_{i} h_{i}$, define:

$$
|\mathcal{H}|_{\mathscr{B}}:=\sum\left|\lambda_{i}\right|
$$

This norm is equivalent to any other norm since $\operatorname{harm}^{1}(M, g)$ is a finite dimensional vector space. Here we choose this one for convenience in the calculations and estimates to come later.

Given $X \in \operatorname{sym} p(M, \omega)$, we consider the Hodge decomposition of $i_{X} \omega$ [14] : there is a unique harmonic 1-form $\mathscr{H}_{X}$ and a unique function $u_{X}$ such that

$$
i_{X} \omega=\mathscr{H}_{X}+d u_{X}
$$

Recall that the function $u_{X}$ is given by the following formula: $u_{X}=\delta G(i(X) \omega)$, where $\delta$ is the codifferential and $G$ is the Green operator (see [14]).

This defines a decompsition of $X \in \operatorname{sym} p(M, \omega)$ as $: X=\# \mathscr{H}_{X}+X_{u_{X}}$, where $\# \mathcal{H}_{X}$ is defined by the equation $i\left(\# \mathscr{H}_{X}\right) \omega=\mathscr{H}_{X}$ and $X_{u_{X}}$ is the hamiltonian vector field with $u_{X}$ as hamiltonnian.

We now define a norm $\|$.$\| on the the vector space \operatorname{sym} p(M, \omega)$ by:

$$
\|X\|=\left|\mathscr{H}_{X}\right|_{\mathscr{B}}+\operatorname{osc}\left(u_{X}\right) .
$$

It is easy to see that this is a norm. Let us just verify that $\|X\|=0$ implies that $X=0$. Indeed $\left|\mathscr{H}_{X}\right|_{\mathscr{B}}=0$ implies that $i_{X} \omega=d u_{X}$, and $\operatorname{osc}\left(u_{X}\right)=0$ implies that $u_{X}$ is a constant, therefore $d u_{X}=0$.

Remark: This norm is not invariant by $\operatorname{Symp}(M, \omega)$. Hence it does not define a Finsler metric on $\operatorname{Symp}(M, \omega)$.

The norm ||.|| defined above depends of course on the riemannian metric $g$ and the basis $\mathscr{B}$ of harmonic 1-forms. However, we have the following:

Theorem 1: All the norms |..| defined by equation (1) using different riemannian metrics and different basis of harmonic 1-forms are equivalent.

Hence the topology on the space symp $(M, \omega)$ of symplectic vector fields defined by the norm (1) is intrinsic: it is independent of the choice of the riemannian metric $g$ and of the basis $\mathscr{B}$ of harmonic 1-forms. 
For each symplectic isotopy $\Phi=\left(\phi_{t}\right)$, consider the Hodge decomposition of $i_{\left(\dot{\phi}_{t}\right)} \omega$

$$
i_{\left(\dot{\phi}_{t}\right)} \omega=\mathscr{H}_{t}^{\Phi}+d u_{t}^{\Phi}
$$

where $\mathscr{H}_{t}^{\Phi}$ is a harmonic 1 -form.

We define the length $l(\Phi)$ of the isotopy $\Phi=\left(\phi_{t}\right)$ by:

$$
l(\Phi)=\int_{0}^{1}\left(\left|\mathscr{H}_{t}^{\Phi}\right|+o s c\left(u_{t}^{\Phi}\right)\right) d t=\int_{0}^{1}\left\|\dot{\phi}_{t}\right\| d t
$$

One also writes

$$
\int_{0}^{1}\left\|\dot{\phi}_{t}\right\| d t=\left\|\dot{\phi}_{t}\right\|
$$

In the expressions above, we have written $\left|\mathscr{H}_{t}^{\Phi}\right|$ for $\left|\mathscr{H}_{t}^{\Phi}\right|_{\mathscr{B}}$, where $\mathscr{B}$ is a fixed basis of $\operatorname{harm}^{1}(M, g)$, for a fixed riemannian metric $g$.

We define the distance $D_{0}(\Phi, \Psi)$ between two symplectic isotopies $\Phi=\left(\phi_{t}\right)$ and $\Psi=\left(\psi_{t}\right)$ by:

$$
D_{0}(\Phi, \Psi)=\left\||| \dot{\phi}_{t}-\dot{\psi}_{t} \mid\right\|:=\int_{0}^{1}\left(\left|\mathscr{H}_{t}^{\Phi}-\mathscr{H}_{t}^{\Psi}\right|+o s c\left(u^{\Phi_{t}}-u^{\Psi_{t}}\right)\right) d t
$$

Denote by $\Phi^{-1}=\left(\phi_{t}^{-1}\right)$ and by $\Psi^{-1}=\left(\psi_{t}^{-1}\right)$ the Inverse isotopies.

\section{Remarks:}

1. The distance $D_{0}(\Phi, \Psi) \neq l\left(\Psi^{-1} \Phi\right)$ unless $\Psi$ and $\Phi$ are hamiltonian isotopies ( see proposition 1).

2. $l(\Phi) \neq l\left(\Phi^{-1}\right)$ unless $\Phi$ is hamiltonian. Indeed, $\mathscr{H}_{t}^{\Phi^{-1}}=-\mathscr{H}_{t}^{\Phi}$ but $u_{t}^{\Phi}$ is very differerent from $u_{t}^{\Phi^{-1}}$. The formula of the difference $u_{t}^{\Phi}-u_{t}^{\Phi^{-1}}$ follows from propositions 3 , 4 and 5 .

In view of the remarks above, we define a more "symmetrical" distance $D$ by:

$$
D(\Phi, \Psi)=\left(D_{0}(\Phi, \Psi)+D_{0}\left(\Phi^{-1}, \Psi^{-1}\right)\right) / 2
$$

Following [13], we define the symplectic distance on $I s o(M, \omega)$ by:

$$
d_{\text {symp }}(\Phi, \Psi)=\bar{d}(\Phi, \Psi)+D(\Phi, \Psi) \text {. }
$$

Definition: The symplectic topology on $\operatorname{Iso}(M, \omega)$ is the metric topology defined by the distance $d_{\text {symp }}$.

Theorem 1': The symplectic topology on Iso $(M, \omega)$ is canonical: it is independent of all choices involved in its definition. 
We may also define another distance $D^{\infty}$ on $I s o(M, \omega)$ :

$$
\begin{gathered}
\left.D_{0}^{\infty}(\Phi, \Psi)=\sup _{t \in[0,1]}\left(\left|\mathscr{H}_{t}^{\Phi}-\mathscr{H}_{t}^{\Psi}\right|\right)+\sup p_{t \in[0,1]} \operatorname{osc}\left(u^{\Phi_{t}}-u^{\Psi_{t}}\right)\right) \\
D^{\infty}(\Phi, \Psi)=\left(D_{0}^{\infty}(\Phi, \Psi)+D_{0}^{\infty}\left(\Phi^{-1}, \Psi^{-1}\right)\right) / 2
\end{gathered}
$$

and

$$
d_{\text {symp }}^{\infty}(\Phi, \Psi)=\bar{d}(\Phi, \Psi)+D^{\infty}(\Phi, \Psi)
$$

Proposition 1: Let $\Phi=\left(\phi_{t}\right), \Psi=\left(\psi_{t}\right)$ be two hamiltonian isotopies and $\sigma_{t}=\left(\psi_{t}\right)^{-1} \phi_{t}$ then

$$
\left\|\left|\dot{\sigma}_{t}\||=|\| \dot{\phi}_{t}-\dot{\psi}_{t} \|\right|=\int_{0}^{1} \operatorname{osc}\left(u_{t}^{\Phi}-u^{\Psi_{t}}\right) d t\right.
$$

Proof: This follows immediately from the equation

$$
\dot{\sigma}_{t}=\left(\psi_{t}^{-1}\right)_{*}\left(\dot{\phi}_{t}-\dot{\psi}_{t}\right)
$$

which is a consequence of proposition 4 stated in section 4 .

Corollary: The distance $d_{\text {sym }}$ reduces to the hamiltonian distance $d_{\text {ham }}$ when $\Phi$ and $\Psi$ are hamiltonian isotopies.

The symplectic topology reduces to the "hamiltonian topology" of [13] on paths in $\operatorname{Ham}(M, \omega)$.

A Hofer-like metric on $\operatorname{Sym} p(M, \omega)_{0}$

For any $\phi \in \operatorname{Sym} p(M, \omega)$, define:

$$
e_{0}(\phi)=\inf (l(\Phi))
$$

where the infimum is taken over all symplectic isotopies $\Phi$ from $\phi$ to the identity. The following result was proved in [2].

Theorem: The map e : $\operatorname{Sym} p(M, \omega)_{0} \rightarrow \mathbb{R} \cup\{\infty\}$ :

$$
e(\phi)=:\left(e_{0}(\phi)+e_{0}\left(\phi^{-1}\right)\right) / 2
$$

is a metric on the identity component $\operatorname{Sym} p(M, \omega)_{0}$ in the group $\operatorname{Symp}(M, \omega)$, i.e. it satisfies

(i) $e(\phi) \geq 0$ and $e(\phi)=0$ iff $\phi$ is the identity.

(ii) $e(\phi)=e\left((\phi)^{-1}\right)$

(iii) $e(\phi . \psi) \leq(e \phi)+e(\psi)$.

The restriction to $\operatorname{Ham}(M, \omega)$ is bounded from above by the Hofer norm. 
Recall that the Hofer norm [10] of a hamiltonian diffeomorphism $\phi$ is

$$
\|\phi\|_{H}=\inf \left(l\left(\Phi_{H}\right)\right)
$$

where the infimum is taken over all hamiltonian isotopies from $\phi$ to the identity.

The Hofer-like metric above depends on the choice of a riemannian metric $g$ and a basis $\mathscr{B}$ of harmonic 1-forms. Hence it is not "natural". However, by theorem 1 , all the metrics constructed that way are equivalent; so they define a natural topology on $\operatorname{Sym} p(M, \omega)_{0}$.

\section{Strong Symplectic Homeomorphisms}

Definition: A homeomorphism $h$ is said to be a strong symplectic homeomorphism if there exists a continuous path $\lambda:[0,1] \rightarrow H o m e o(M)$ such that $\lambda(0)=i d ; \lambda(1)=h$ and a sequence $\Phi^{n}=\left(\phi_{t}^{n}\right)$ of symplectic isotopies, which converges to $\lambda$ in the $C^{0}$ topology (induced by the norm $\bar{d})$ and such that $\Phi^{n}$ is Cauchy for the metric $d_{\text {symp. }}$.

We will denote by $S S y m p e o(M, \omega)$ the set of all strong symplectic homeomorphisms. This set is well defined independently of any riemannian metric or any basis of harmonic 1-forms.

Clearly, if $M$ is simply connected, the set $\operatorname{SSympeo}(M, \omega)$ coincides with the group $\operatorname{Hameo}(M, \omega)$.

We denote by $S \operatorname{Sympeo}(M, \omega)^{\infty}$ the set defined like in $S \operatorname{Sympeo}(M, \omega)$ but replacing the norm $d_{\text {symp }}$ by the norm $d_{\text {symp }}^{\infty}$.

Let $\mathscr{P H o m e o}(M)$ be the set of continuous paths $\gamma:[0,1] \rightarrow H o m e o(M)$ such that $\gamma(0)=$ $i d$, and let $\mathscr{P}^{\infty}\left(\operatorname{Harm}^{1}(M)\right.$ be the space of smooth paths of harmonic 1-forms.

We have the following maps:

$$
\begin{aligned}
& A_{1}: I s o(M, \omega) \rightarrow \mathscr{P H o m e o}(M), \Phi \mapsto \Phi(t) \\
& A_{2}: I s o(M, \omega) \rightarrow \mathscr{P}^{\infty}\left(\operatorname{Harm}^{1}(M), \Phi \mapsto \mathcal{H}_{t}^{\Phi}\right. \\
& A_{3}: I s o(M, \omega) \rightarrow C^{\infty}(M \times[0,1], \mathbb{R}), \Phi \mapsto u^{\Phi}
\end{aligned}
$$

Let $\mathscr{Q}$ be the image of the mapping $A=A_{1} \times A_{2} \times A_{3}$ and $\overline{\mathscr{Q}}$ the closure of $\mathscr{Q}$ inside $\mathscr{I}(M, \omega)=: \mathscr{P H o m e o}(M) \times \mathscr{P}^{\infty}\left(\operatorname{Harm}^{1}(M) \times C^{\infty}(M \times[0,1], \mathbb{R})\right.$, with the symplectic topology, which is the $C^{0}$ topology on the first factor and the metric topology from $D$ on the second and third factor

Then $S S y m p e o(M, \omega)$ is just the image of the evaluation map of the path at $\mathrm{t}=1$ of the image of the projection of $\overline{\mathscr{Q}}$ on the first factor. This defines a surjective map:

$$
a: \overline{\mathscr{Q}} \rightarrow \operatorname{SSympeo}(M, \omega)
$$


The symplectic topology on $S \operatorname{Sympeo}(M, \omega)$ is the quotient topology induced by $a$.

Our main results are :

Theorem 2: The set $\overline{\mathscr{Q}}$ is a topological group.

Theorem 3: Let $(M, \omega)$ be a closed symplectic manifold. Then SSympeo $(M, \omega)$ is an arcwise connected topological group (with the sympectic topology), containing $\operatorname{Hameo}(M, \omega)$ as a normal subgroup, and contained in the path component of the identity $\operatorname{Sympeo}_{0}(M, \omega)$ of Sympeo $(M, \omega)$.

If $M$ is simply connected, $S \operatorname{Sympeo}(M, \omega)=\operatorname{Hameo}(M, \omega)$. Finally, the commutator subgroup $[S \operatorname{Sympeo}(M, \omega), \operatorname{SSympeo}(M, \omega)]$ of SSympeo $(M, \omega)$ is contained in Hameo $(M, \omega)$.

\section{Conjectures:}

1. Let $(M, \omega)$ be a closed symplectic manifold, then $[\operatorname{SSympeo}(M, \omega), \operatorname{SSympeo}(M, \omega)]=$ Hameo $(M, \omega)$.

2. The inclusion $\operatorname{SSympeo}(M, \omega) \subset \operatorname{Sympeo}_{0}(M, \omega)$ is strict.

3. The results in theorem 3 hold for $\operatorname{SSympeo}(M, \omega)^{\infty}$.

Conjecture 3 is supported by a result of Muller asserting that $\operatorname{Hameo}(M, \omega)$ coincides with $\operatorname{Hameo}(M, \omega)^{\infty}$ which is defined by replacing the $L^{(1, \infty)}$ Hofer norm by the $L^{\infty}$ norm [12].

\section{Measure preserving homeomorphisms}

On a symplectic $2 n$ dimensional manifold $(M, \omega)$, we consider the measure $\mu_{\omega}$ defined by the Liouville volume $\omega^{n}$. Let $\operatorname{Homeo}_{0}^{\mu_{\omega}}(M)$ be the identity component in the group of homeomorphisms preserving $\mu_{\omega}$. We have:

$$
\operatorname{Sympeo}_{0}(M, \omega) \subset \operatorname{Homeo}_{0}^{\mu_{\omega}}(M) .
$$

Oh and Müller [13] have observed that $\operatorname{Hameo}(M, \omega)$ is a sub-group of the kernel of Fathi's mass-flow homomorphism [8]. This is a homomorphism $\theta: \operatorname{Homeo}_{0}^{\mu_{\omega}}(M) \rightarrow H_{1}(M, \mathbb{R}) / \Gamma$, where $\Gamma$ is some sub-group of $H_{1}(M, \mathbb{R})$. Fathi proved that if the dimension of $M$ is bigger than 2, then $\operatorname{Ker} \theta$ is a simple group. This leaves open the following question [13]:

$$
\text { Is } \operatorname{Homeo}_{0}^{\mu_{\omega}}\left(S^{2}\right)=\operatorname{Sympeo}_{0}\left(S^{2}, \omega\right) \text { a simple group? }
$$

But $\operatorname{Sympeo} 0\left(S^{2}, \omega\right)$ contains $\operatorname{Hameo}\left(S^{2}, \omega\right)$ as a normal subgroup. The question is to decide if the inclusion

$$
\operatorname{Hameo}\left(S^{2}, \omega\right) \subset \operatorname{Sympeo}_{0}\left(S^{2}, \omega\right)
$$


is strict. Since $\operatorname{SSympeo}\left(S^{2}, \omega\right)=\operatorname{Hameo}\left(S^{2}, \omega\right)$, our conjecture 2 implies that $\operatorname{Homeo}_{0}^{\mu_{\omega}}\left(S^{2}\right)=$ Sympeo $_{0}\left(S^{2}, \omega\right)$ is not a simple group, a conjecture of [13].

\section{Questions}

1. Is $\operatorname{SSympeo}(M, \omega)$ a normal subgroup of $\operatorname{Sympeo} 0(M, \omega)$ ?

2. Is $\left[\operatorname{Sympeo}_{0}(M, \omega), \operatorname{Sympeo}_{0}(M, \omega)\right]$ contained in $\operatorname{Hameo}(M, \omega)$ ?

\section{Proofs of the Results}

\subsection{Proof of theorem 1}

If $\mathscr{B}$ and $\mathscr{B}^{\prime}$ are two basis of $\operatorname{harm}^{1}(M, g)$, then elementary linear algebra shows that $|.|_{\mathscr{B}}$ and $|.|_{\mathscr{B}^{\prime}}$ are equivalent. This implies that the corresponding norms on $\operatorname{sym} p(M, \omega)$ are also equivalent.

Let us now start our construction with a riemannian metric $g$ and a basis $\mathscr{B}=\left(h_{1}, . . h_{k}\right)$ of $\operatorname{harm}^{1}(M, g)$. We saw that for any $X \in \operatorname{symp}(M, \omega)$,

$$
i_{X} \omega=\mathscr{H}_{X}+d u_{X}
$$

and we wrote $\mathscr{H}_{X}=\sum \lambda_{i} h_{i}$.

Let $g^{\prime}$ be another riemannian metric. The $g^{\prime}$-Hodge decomposition of $i_{X} \omega$ is:

$$
i_{X} \omega=\mathscr{H}_{X}^{\prime}+d u_{X}^{\prime}
$$

where $\mathscr{H}_{X}^{\prime}$ is $g^{\prime}$-harmonic.

Consider the $g^{\prime}$-Hodge decompositions of the members $h_{i}$ of the basis $\mathscr{B}$ i.e.

$$
h_{i}=h_{i}^{\prime}+d v_{i}
$$

where $h_{i}^{\prime}$ is $g^{\prime}$ harmonic. $\mathscr{B}^{\prime}=\left(h_{1}^{\prime}, . . h_{k}^{\prime}\right)$ is a basis of $\operatorname{harm}^{1}\left(M, g^{\prime}\right)$. Indeed suppose that $\sum r_{i} h_{i}^{\prime}=0$. The 1-form $\sum r_{i} h_{i}=d\left(\sum r_{i} v_{i}\right)$ is $g$-harmonic and exact $: \sum r_{i} h_{i}=d\left(\sum r_{i} v_{i}\right)$. But an exact harmonic form must be identically zero. Therefore all $r_{i}$ are zero since $\left\{h_{i}\right\}$ form a basis. Hence $\left\{h_{i}^{\prime}\right\}$ are linearly independent.

The 1-form

$$
\mathscr{H}_{X}^{\prime \prime}=: \sum \lambda_{i} h_{i}^{\prime}
$$

is a $g^{\prime}$ - harmonic form representing the cohomology class of $i_{X} \omega$. By uniqueness, $\mathscr{H}_{X}^{\prime}=\mathscr{H}_{X}^{\prime \prime}$.

Hence

$$
\left|\mathscr{H}_{X}^{\prime}\right|_{\mathscr{B}}=\sum\left|\lambda_{i}\right|=\left|H_{X}\right|_{\mathscr{B}}
$$


Furthermore $\mathscr{H}_{X}^{\prime}=\sum \lambda_{i}\left(h_{i}-d v_{i}\right)=\mathscr{H}_{X}+d v$ where $v=-\sum \lambda_{i} v_{i}$. Hence

$$
i_{X} \omega=\mathscr{H}_{X}^{\prime}+d u_{X}^{\prime}=\mathscr{H}_{X}+d\left(v+u_{X}^{\prime}\right)
$$

By uniqueness in the $g$-Hodge decomposition of $i_{X} \omega$,

$$
u_{X}=v+u_{X}^{\prime} .
$$

Denote by $\|X\|_{g^{\prime}}$, resp. $\|X\|_{g}$, the norm of $X$ using the riemannian metric $g^{\prime}$ and the basis $\mathscr{B}^{\prime}$, resp. using the riemannian metric $g$ and the basis $\mathscr{B}$. Then:

$$
\begin{gathered}
\|X\|_{g^{\prime}}=\left|\mathscr{H}_{X}^{\prime}\right|_{\mathscr{B}^{\prime}}+\operatorname{osc}\left(u_{X}^{\prime}\right)=\left|\mathscr{H}_{X}^{\prime}\right|_{\mathscr{B}^{\prime}}+\operatorname{osc}\left(u_{X}-v\right) \\
\leq\left|\mathscr{H}_{X}^{\prime}\right|_{\mathscr{B}^{\prime}}+\operatorname{osc}\left(u_{X}\right)+\operatorname{osc}(-v) \\
=\left|\mathscr{H}_{X}\right|_{\mathscr{B}}+\operatorname{osc}\left(u_{X}\right)+\operatorname{osc}(v)=\|X\|_{g}+o s c(v) .
\end{gathered}
$$

Let $c=2 \max _{i}\left|v_{i}\right|$, since $v=-\sum \lambda_{i} v_{i}$, we get the following inequality:

$$
\operatorname{osc}(v) \leq 2 \max (|v|) \leq c\left|\mathscr{H}_{X}\right|_{\mathscr{B}}=c\left|\mathscr{H}_{X}^{\prime}\right|_{\mathscr{B}}
$$

Therefore

$$
\|X\|_{g^{\prime}} \leq\|X\|_{g}+o s c(v) \leq\|X\|_{g}+c\left|\mathscr{H}_{X}\right|_{\mathscr{B}} \leq\|X\|_{g}+c\left(\left|\mathscr{H}_{X}\right|_{\mathscr{B}}+o s c\left(u_{X}\right)\right)=(c+1)\|X\|_{g}
$$

Similarly,

$$
\begin{aligned}
\|X\|_{g}= & \left|\mathscr{H}_{X}\right|_{\mathscr{B}}+\operatorname{osc}\left(u_{X}\right)=\left|\mathscr{H}_{X}\right|_{\mathscr{B}}+\operatorname{osc}\left(u_{X}^{\prime}+v\right) \leq\left|\mathscr{H}_{X}\right|_{\mathscr{B}}+\operatorname{osc}\left(u_{X}^{\prime}\right)+\operatorname{osc}(v) \\
= & \left|\mathscr{H}_{X}^{\prime}\right|_{\mathscr{B}^{\prime}}+\operatorname{osc}\left(u_{X}^{\prime}\right)+\operatorname{osc}(v)=\|X\|_{g^{\prime}}+\operatorname{osc}(v) \leq\|X\|_{g^{\prime}}+c\left|\mathcal{H}_{X}^{\prime}\right|_{\mathscr{B}^{\prime}} \\
& \leq\|X\|_{g^{\prime}}+c\left(\left|\mathscr{H}_{X}^{\prime}\right|_{\mathscr{B}^{\prime}}+\operatorname{osc}\left(u_{X}^{\prime}\right)=(c+1)\|X\|_{g^{\prime}}\right.
\end{aligned}
$$

Hence the metrics $\|X\|_{g}$ and $\|X\|_{g}$ are equivalent

For the purpose of the proof of the main theorem, we fix a riemannian metric $g$ and a basis $\mathscr{B}=\left(h_{1}, . ., h_{k}\right)$ of $\operatorname{harm}^{1}(M, g)$. The norm of a harmonic 1 -form $\mathscr{H}$ will be simply denoted $|\mathscr{H}|$ and the norm of a symplectic vector field $X$ will be simply denoted $\|X\|$.

\subsection{Proof of theorem 3}

We prove first that the set $\operatorname{SSympeo}(M, \omega)$ subsetSympeo $(M, \omega)$ is closed under composition and inverse maps.

Let $h_{i} \in S \operatorname{Sympeo}(M, \omega) i=1,2$ and let $\lambda_{i}$ be continuous paths in Homeo(M) with $\lambda_{i}(0)=i d, \lambda_{i}(1)=h_{i}$ and let $\Phi_{i}^{n}$ be $d_{\text {symp }}$ - Cauchy sequences of symplectic isotopies converging $C^{0}$ to $\lambda_{i}$. Then $\Phi_{1}^{n} .\left(\Phi_{2}^{n}\right)^{-1}$ converges $C^{0}$ to the path $\lambda_{1}(t)\left(\lambda_{2}(t)\right)^{-1}$. Here $\Phi_{1}^{n} \cdot\left(\Phi_{2}^{n}\right)^{-1}(t)=$ $\phi_{1}^{n}(t) \cdot\left(\phi_{2}^{n}(t)\right)^{-1}$. 
By definition of the distance $d_{\text {symp }}, \Phi^{n}$ is a $d_{\text {symp }}$ - Cauchy sequence if and only if both $\Phi^{n}$ and $\left(\Phi^{n}\right)^{-1}$ are $D_{0}$ - Cauchy and $\bar{d}$ - Cauchy sequences.

Main Lemma: If $\Phi^{n}=\left(\phi_{t}^{n}\right)$ and $\Psi_{t}^{n}=\left(\psi_{t}^{n}\right)$ are $d_{\text {symp }}$ - Cauchy sequences in Iso(M), so is $\rho_{t}^{n}=\phi_{t}^{n} \psi_{t}^{n}$.

The proof of the main lemma is very delicate; it will take most of the remaining part of this paper. The estimates are much more involved than in the hamiltonian case, due to the fact that the decomposition of a symplectic isotopy into a hamiltonian one and a harmonic one does not behave nicely with respect to the product of isotopies.

It will be enough to prove that $\rho_{t}^{n}$ is a $D_{0}$ - Cauchy sequence. Indeed since $\left(\Phi^{n}\right)^{-1}$ and $\left(\Psi^{n}\right)^{-1}$ are $D_{0}$ - Cauchy by assumption, the main lemma applied to their product implies that their product is also $D_{0}$ Cauchy.

Hence $\left(\Psi^{n}\right)^{-1}\left(\Phi^{n}\right)^{-1}=\left(\Phi^{n} \Psi^{n}\right)^{-1}=\left(\rho_{t}^{n}\right)^{-1}$ is a $D_{0}$ - Cauchy sequence. This will conclude the proof that $S \operatorname{Sympeo}(M, \omega)$ is a group. We leave the details to the reader.

We will use the following estimate:

Proposition 2: There exists a constant $E$ such that for any $X \in \operatorname{symp}(M, \omega)$, and $\mathscr{H} \in$ $\operatorname{harm}^{1}(M, g)$

$$
|\mathscr{H}(X)|=: \sup _{x \in M}|\mathscr{H}(x)(X(x))| \leq E|| X|| .|\not{H}|
$$

Proof: Let $\left(h_{1}, . ., h_{r}\right)$ be the chosen basis for harmonic 1-forms and let $E=\max _{i} E_{i}$ and $E_{i}=$ $\sup _{V}\left(\sup _{x \in M}\left|h_{i}(x)(V(x))\right|\right.$ where $V$ runs over all symplectic vector fields $V$ such that $\|V\|=1$.

Without loss of generality, we may suppose $X \neq 0$ and set $V=X /\|X\|$. Let $\mathscr{H}=\sum \lambda_{i} h_{i}$. Then $\mathscr{H}(X)=\|X\| \sum \lambda_{i} h_{i}(V)$. Hence

$$
|\not{H}(X)| \leq\|X\| \sum\left|\lambda_{i}\right| \sup _{x}\left(\left|h_{i}(x)(V)(x)\right|\right) \leq\|X\| \sum\left|\lambda_{i}\right| E=E\|X\| .|\not \mathscr{C}| .
$$

We will also need the following standard facts:

Proposition 3: Let $\phi$ be a diffeomorphism, $X$ a vector field and $\theta$ a differential form on a smooth manifold $M$. Then

$$
\left(\phi^{-1}\right)^{*}\left[i_{X} \phi^{*} \theta\right]=i_{\phi_{*} X} \theta
$$

Proposition 4: If $\phi_{t}, \psi_{t}$ are any isotopies, and if we denote by $\rho_{t}=\phi_{t} \psi_{t}$, and by $\underline{\phi}_{t}=(\phi)_{t}^{-1}$ then

$$
\dot{\rho}_{t}=\dot{\phi}_{t}+\left(\phi_{t}\right)_{*} \dot{\psi}_{t}
$$


and

$$
\dot{\phi}_{t}=-\left((\phi)_{t}^{-1}\right)_{*}\left(\dot{\phi}_{t}\right)
$$

Proposition 5: Let $\theta_{t}$ be a smooth family of closed 1-forms and $\phi_{t}$ an isotopy, then

$$
\phi_{t}^{*} \theta_{t}-\theta_{t}=d v_{t}
$$

where

$$
v_{t}=\int_{0}^{t}\left(\theta_{t}\left(\dot{\phi}_{s}\right) \circ \phi_{s}\right) d s
$$

Proof of the Main Lemma: If $\phi_{t}, \psi_{t}$ are symplectic isotopies, and if $\rho_{t}=\phi_{t} \psi_{t}$, propositions 3,4 and 5 give:

$$
i\left(\dot{\rho}_{t}\right) \omega=\mathscr{H}_{t}^{\Phi}+\mathscr{H}_{t}^{\Psi}+d K(\Phi, \Psi)
$$

where $K=K(\Phi, \Psi)=u_{t}^{\Phi}+\left(u_{t}^{\Psi}\right) \circ\left(\phi_{t}\right)^{-1}+v_{t}(\Phi, \Psi)$, and

$$
v_{t}(\Phi, \Psi)=\int_{0}^{t}\left(\mathscr{H}_{t}^{\Psi}\left(\dot{\phi}_{s}\right) \circ \phi_{s}^{-1}\right) d s .
$$

Let now $\phi_{t}^{n}, \psi_{t}^{n}$ be Cauchy sequences of symplectic isotopies, and consider the sequence $\rho_{t}^{n}=\phi_{t}^{n} \psi_{t}^{n}$.

We have:

$$
\begin{gathered}
\left\|\dot{\rho}_{t}^{n}-\dot{\rho}_{t}^{m}\left|\|=\int_{0}^{1}\right| \mathscr{H}_{t}^{\Phi^{n}}-\mathscr{H}_{t}^{\Phi^{m}}+\mathscr{H}_{t}^{\Psi^{n}}-\mathscr{H}_{t}^{\Psi^{m}} \mid+\operatorname{osc}\left(K\left(\Phi^{n}, \Psi^{n}\right)-K\left(\Phi^{m}, \Psi^{m}\right)\right) d t\right. \\
\left.\left.\leq \int_{0}^{1} \mid \mathscr{H}_{t}^{\Phi^{n}}-\mathscr{H}_{t}^{\Phi^{m}}\right)\left|d t+\int_{0}^{1}\right| \mathscr{H}_{t}^{\Psi^{n}}-\mathscr{H}_{t}^{\Psi^{m}}\right) \mid d t \\
\left.+\int_{0}^{1} \operatorname{osc}\left(u_{t}^{\Phi^{n}}-u_{t}^{\Phi^{m}}\right) d t+\int_{0}^{1} \operatorname{osc}\left(u_{t}^{\Psi^{n}}\right) \circ\left(\phi_{t}^{n}\right)^{-1}-u_{t}^{\Psi^{m}} \circ\left(\phi_{t}^{m}\right)^{-1}\right) d t \\
\quad+\int_{0}^{1} \operatorname{osc}\left(v_{t}\left(\Phi^{n}, \Psi^{n}\right)-v_{t}\left(\Phi^{m}, \Psi^{m}\right) d t\right. \\
\left.=\left\|\phi^{n}{ }_{t}-\phi^{m}{ }_{t}\right\|\left|+\int_{0}^{1}\right| \mathcal{H}_{t}^{\Psi^{n}}-\mathscr{H}_{t}^{\Psi^{m}}\right) \mid d t+A+B
\end{gathered}
$$

where

$$
\left.A=\int_{0}^{1} \operatorname{osc}\left(u_{t}^{\Psi^{n}}\right) \circ\left(\phi_{t}^{n}\right)^{-1}-u_{t}^{\Psi^{m}} \circ\left(\phi_{t}^{m}\right)^{-1}\right) d t
$$

and

$$
B=\int_{0}^{1} \operatorname{osc}\left(v_{t}\left(\Phi^{n}, \Psi^{n}\right)-v_{t}\left(\Phi^{m}, \Psi^{m}\right) d t\right.
$$


We have:

$$
\begin{gathered}
\left.\left.A \leq \int_{0}^{1} \operatorname{osc}\left(u_{t}^{\Psi^{n}}\right) \circ\left(\phi_{t}^{n}\right)^{-1}-u_{t}^{\Psi^{m}} \circ\left(\phi_{t}^{n}\right)^{-1}\right) d t+\int_{0}^{1} \operatorname{osc}\left(u_{t}^{\Psi^{m}}\right) \circ\left(\phi_{t}^{n}\right)^{-1}-\left(u_{t}^{\Psi^{m}}\right) \circ\left(\phi_{t}^{m}\right)^{-1}\right) d t \\
=\int_{0}^{1} \operatorname{osc}\left(u_{t}^{\Psi^{n}}-u_{t}^{\Psi^{m}}\right) d t+C
\end{gathered}
$$

where

$$
C=\int_{0}^{1} \operatorname{osc}\left(u_{t}^{\Psi^{m}} \circ\left(\phi_{t}^{n}\right)^{-1}-u_{t}^{\Psi^{m}} \circ\left(\phi_{t}^{m}\right)^{-1}\right) d t
$$

Hence

$$
\begin{gathered}
\left|\left\|\dot{\rho}_{t}^{n}-\dot{\rho}_{t}^{m}\left|\left\|\leq\left|\left\|\dot{\phi}_{t}^{n}-\dot{\phi}_{t}^{m}|\||\right.\right.\right.\right.\right.\right. \\
\left.+\int_{0}^{1} \mid \mathscr{H}_{t}^{\Psi^{n}}-\mathscr{H}_{t}^{\Psi^{m}}\right) \mid d t+\int_{0}^{t} \operatorname{osc}\left(u_{t}^{\Psi^{n}}-u_{t}^{\Psi^{m}}\right) d t+B+C \\
=\left\|\dot{\phi}_{t}^{n}-\dot{\phi}_{t}^{m}\left|\left\||+||| \dot{\psi}_{t}^{n}-\dot{\psi}_{t}^{m} \mid\right\|+B+C\right.\right.
\end{gathered}
$$

We now show that $C \rightarrow 0$ when $m, n \rightarrow \infty$.

Sub-Lemma 1 (Reparametrization Lemma [13]): $\forall \epsilon \geq 0, \exists m_{0}$ such that

$$
\left.C=\int_{0}^{1} \operatorname{osc}\left(u_{t}^{\Psi^{m}} \circ\left(\phi_{t}^{n}\right)^{-1}-u_{t}^{\Psi^{m}} \circ\left(\phi_{t}^{m}\right)^{-1}\right) d t=: \| u_{t}^{\Psi^{m}} \circ\left(\phi_{t}^{n}\right)^{-1}-u_{t}^{\Psi^{m}} \circ\left(\phi_{t}^{m}\right)^{-1}\right) \| \leq \epsilon
$$

if $m \geq m_{0}$ and $n$ large enough

Remark: This is the "reparametrization lemma" of Oh-Müller [13] (lemma 3.21. (2)). For the convenience of the reader and further references, we include their proof.

Proof: For short, we write $u_{m}$ for $u_{t}^{\Psi^{m}}$ and $\mu_{t}^{n}$ for $\left(\phi_{t}^{n}\right)^{-1}$.

First, there exists $m_{0}$ large such that $\left\|u_{m}-u_{m_{0}}\right\| \leq \epsilon / 3$ for $m \geq m_{0}$, since $\left(u_{m}\right)$ is a Cauchy sequence for the distance $d\left(u_{n}, u_{m}\right)=\int_{0}^{1}$ osc $\left(u_{n}-u_{m}\right) d t$.

Therefore

$$
\begin{gathered}
\left.\left.\left.\left.\left.\left.\left.\left.\| u_{m} \circ \mu_{t}^{n}-u_{m} \circ \mu_{t}^{m}\right)\right)\|\leq\| u_{m} \circ \mu_{t}^{n}-u_{m_{0}} \circ \mu_{t}^{n}\right)\right)\|+\| u_{m_{0}} \circ \mu_{t}^{n}-u_{m_{0}} \circ \mu_{t}^{m}\right)\right)\|+\| u_{m_{0}} \circ \mu_{t}^{m}-u_{m} \circ \mu_{t}^{m}\right)\right) \| \\
\left.\left.=\left\|u_{m}-u_{m_{0}}\right\|+\| u_{m_{0}} \circ \mu_{t}^{n}-u_{m_{0}} \circ \mu_{t}^{m}\right)\right)\|+\| u_{m_{0}}-u_{m} \| \\
\left.\left.\leq(2 / 3) \epsilon+\| u_{m_{0}} \circ \mu_{t}^{n}-u_{m_{0}} \circ \mu_{t}^{m}\right)\right) \mid .
\end{gathered}
$$

By uniform continuity of $u_{m_{0}}$, there exists a positive $\delta$ such that if $\bar{d}\left(\mu_{t}^{m}, \mu_{t}^{n}\right) \leq \delta$, then $\max \operatorname{osc}\left(\left(u_{m_{0}} \circ \mu_{t}^{n}-u_{m_{0}} \circ \mu_{t}^{m}\right)\right) \leq \epsilon / 3$. Hence $\left.\left.\| u_{m_{0}} \circ \mu_{t}^{n}-u_{m_{0}} \circ \mu_{t}^{m}\right)\right) \| \leq \epsilon / 3$ for $n, m$ large. Recall that $\mu_{t}^{n}$ is a $\bar{d}$ - Cauchy sequence. 
To show that $\dot{\rho}_{t}^{n}$ is a Cauchy sequence, the only thing which is left is to show that $B \rightarrow 0$ when $n, m \rightarrow \infty$.

Let us denote $v_{t}\left(\Phi^{n}, \Psi^{n}\right)$ by $v_{t}^{n}, \mathscr{H}_{t}^{\Psi^{n}}$ by $\mathscr{X}_{n}^{t}$ or $\mathscr{H}_{n}$ and $\left(\phi_{t}^{n}\right)^{-1}$ by $\mu_{t}^{n}$.

For a function on $M$, we consider the norm

$$
|f|=\sup _{x \in M}|f(x)|
$$

We have:

$$
\begin{aligned}
\left|v_{t}^{n}-v_{t}^{m}\right| & =\left|\int_{0}^{t}\left(\mathscr{H}_{n}^{t}\left(\dot{\mu}_{s}^{n}\right) \circ \mu_{s}^{n}-\mathscr{H}_{m}^{t}\left(\dot{\mu}_{s}^{m}\right) \circ \mu_{s}^{m}\right) d s\right| \\
& \leq \int_{0}^{1}\left|\left(\left(\mathscr{H}_{n}^{t}-\mathscr{H}_{m}^{t}\right)\left(\dot{\mu}_{s}^{n}\right)\right) \circ \mu_{s}^{n}\right| d s \\
& \left.+\int_{0}^{1} \mid \mathcal{H}_{m}^{t}\left(\dot{\mu}_{s}^{n}-\dot{\mu}_{s}^{m}\right)\right) \circ \mu_{s}^{m} \mid d s \\
+ & \int_{0}^{1}\left|\mathscr{H}_{m}^{t}\left(\dot{\mu}_{s}^{n}\right) \circ \mu_{s}^{n}-\mathscr{H}_{m}^{t}\left(\dot{\mu}_{s}^{n}\right) \circ \mu_{s}^{m}\right| d s
\end{aligned}
$$

The last integral can be estimated as follows:

$$
\begin{aligned}
& \int_{0}^{1}\left|\mathcal{H}_{m}^{t}\left(\dot{\mu}_{s}^{n}\right) \circ \mu_{s}^{n}-\mathscr{H}_{m}^{t}\left(\dot{\mu}_{s}^{n}\right) \circ \mu_{s}^{m}\right| d s \\
\leq & \int_{0}^{1}\left|\mathscr{H}_{m}^{t}\left(\dot{\mu}_{s}^{n}\right) \circ \mu_{s}^{n}-\mathscr{H}_{m}^{t}\left(\dot{\mu}_{s}^{n_{0}}\right) \circ \mu_{s}^{n}\right| d s \\
+ & \int_{0}^{1}\left|\mathcal{H}_{m}^{t}\left(\dot{\mu}_{s}^{n_{0}}\right) \circ \mu_{s}^{n}-\mathscr{H}_{m}^{t}\left(\dot{\mu}_{s}^{n_{0}}\right) \circ \mu_{s}^{m}\right| d s \\
+ & \int_{0}^{1}\left|\mathcal{H}_{m}^{t}\left(\dot{\mu}_{s}^{n_{0}}\right) \circ \mu_{s}^{m}-\mathscr{H}_{m}^{t}\left(\dot{\mu}_{s}^{n}\right) \circ \mu_{s}^{m}\right| d s
\end{aligned}
$$

for some integer $n_{0}$.

Proposition 2 gives $E\left|\mathscr{H}_{m}\right| D_{0}\left(\left(\Phi^{n}\right)^{-1},\left(\Phi^{n_{0}}\right)^{-1}\right) \leq 2 E\left|\mathscr{H}_{m}\right| D\left(\left(\Phi^{n}\right),\left(\Phi^{n_{0}}\right)^{-1}\right)$ as an upper bound for (1) and (3).

It also gives the following estimates:

$$
\begin{gathered}
\left.\int_{0}^{1}\left|\left(\left(\mathscr{H}_{n}^{t}-\mathscr{H}_{m}^{t}\right)\left(\dot{\mu}_{s}^{n}\right)\right) \circ \mu_{s}^{n}\right| d s \leq E\left|\mathscr{H}_{n}^{t}-\mathscr{H}_{m}^{t}\right| \int_{0}^{1}|| \dot{\mu}_{s}^{n}\right)|| d s \\
=E .\left|\mathscr{H}_{n}^{t}-\mathscr{H}_{m}^{t}\right| \cdot l\left(\left(\left(^{n}\right)^{-1}\right)\right.
\end{gathered}
$$


and

$$
\begin{gathered}
\int_{0}^{1}\left|\left(\mathscr{H}_{m}^{t}\left(\dot{\mu}_{s}^{n}-\dot{\mu}_{s}^{m}\right)\right) \circ \mu_{s}^{m}\right| d s \leq E .\left|\mathscr{H}_{m}^{t}\right| \int_{0}^{1}||\left(\dot{\mu}_{s}^{n}-\dot{\mu}_{s}^{m}\right)|| d s \\
\left.\quad=E\left|\mathscr{H}_{m}^{t}\right| D_{0}\left(\left(\Phi^{n}\right)^{-1},\left(\Phi^{m}\right)\right)^{-1}\right) \leq 2 E\left|\mathcal{H}_{m}^{t}\right| D\left(\Phi^{n}, \Phi^{m}\right) .
\end{gathered}
$$

Therefore, we get the following estimate:

$$
\left.\left|v_{t}^{n}-v_{t}^{m}\right| \leq E .\left|\mathcal{H}_{n}^{t}-\mathscr{H}_{m}^{t}\right| l\left(\Phi^{n}\right)^{-1}\right)+E\left|\mathscr{H}_{m}^{t}\right| 2\left(D\left(\Phi^{n}, \Phi^{m}\right)+2 D\left(\Phi^{n}, \Phi^{n_{0}}\right)\right)+G
$$

where

$$
G=\int_{0}^{1}\left|\mathscr{H}_{m}^{t}\left(\dot{\mu}_{s}^{n_{0}}\right) \circ \mu_{s}^{n}-\mathscr{H}_{m}^{t}\left(\dot{\mu}_{s}^{n_{0}}\right) \circ \mu_{s}^{m}\right| d s
$$

Since $\operatorname{osc}\left(v_{t}^{n}-v_{t}^{m}\right) \leq 2\left|v_{t}^{n}-v_{t}^{m}\right|$, we see that

$$
\begin{gathered}
\int_{0}^{1} \operatorname{osc}\left(v_{t}^{n}-v_{t}^{m}\right) d t \leq 2 E\left(l\left(\Phi^{n}\right)^{-1}\right) \int_{0}^{1}\left|\mathcal{H}_{n}^{t}-\mathscr{H}_{m}^{t}\right| d t \\
+E 2\left(D\left(\Phi^{m}, \Phi^{n}\right)+2 E D\left(\Phi^{n}, \Phi^{n_{0}}\right) \int_{0}^{1}\left|\mathcal{H}_{m}^{t}\right| d t\right)+\int_{0}^{1} G d t
\end{gathered}
$$

We need the following facts:

Sub-Lemma 2 (Reparametrization Lemma): $\forall \epsilon \geq 0, \exists n_{0}$ such that

$$
L=\int_{0}^{1} G d t=\int_{0}^{1}\left(\int_{0}^{1}\left|\mathscr{H}_{m}^{t}\left(\dot{\mu}_{s}^{n_{0}}\right) \circ \mu_{s}^{n}-\mathscr{H}_{m}^{t}\left(\dot{\mu}_{s}^{n_{0}}\right) \circ \mu_{s}^{m}\right| d s\right) d t \leq \epsilon
$$

for $n \geq n_{0}$ and $m$ sufficiently large.

Proposition 6: $l\left(\left(\Phi^{n}\right)\right)^{-1}$ and $\int_{0}^{1}\left|\mathcal{H}_{m}^{t}\right| d t$ are bounded for every $n, m$.

We finish first the estimate for $\int_{0}^{1}$ osc $\left(v_{t}^{n}-v_{t}^{m}\right) d t$ using sub-lemma 2 and proposition 6 .

Putting together all the information we gathered, we see that:

$$
\begin{gathered}
\int_{0}^{1} \operatorname{osc}\left(v_{t}^{n}-v_{t}^{m}\right) d t \leq 2 E\left(l\left(\Phi^{n}\right)^{-1}\right) \int_{0}^{1}\left|\mathcal{H}_{n}^{t}-\mathscr{H}_{m}^{t}\right| d t \\
+E\left(2 D\left(\Phi^{m}, \Phi^{n}\right)\right)+2 E D\left(\Phi^{n}, \Phi^{n_{0}}\right)\left(\int_{0}^{1}\left|\mathcal{H}_{m}^{t}\right| d t\right)+L \\
\leq 2 E l\left(\left(\Phi^{n}\right)^{-1}\right) D\left(\Phi^{n}, \Phi^{m}\right)+E\left(2 D\left(\Phi^{m}, \Phi^{n}\right)+2 E D\left(\Phi^{n}, \Phi^{n_{0}}\right) \int_{0}^{1}\left|\mathcal{H}_{m}^{t}\right| d t+L\right.
\end{gathered}
$$

Therefore:

$$
\int_{0}^{1} \operatorname{osc}\left(v_{t}^{n}-v_{t}^{m}\right) d t \rightarrow 0
$$


when $n, m \rightarrow \infty$, and $n_{0}$ is chosen sufficiently large Now let $n_{0} \rightarrow \infty$ as well.. This finishes the proof of the main lemma.

\section{Proof of Sub-Lemma 2:}

$$
\begin{aligned}
G & =\int_{0}^{1}\left|\mathcal{H}_{m}^{t}\left(\dot{\mu}_{s}^{n_{0}}\right) \circ \mu_{s}^{n}-\mathcal{H}_{m}^{t}\left(\dot{\mu}_{s}^{n_{0}}\right) \circ \mu_{s}^{m}\right| d s \\
& \leq \int_{0}^{1}\left|\mathscr{H}_{m}^{t}\left(\dot{\mu}_{s}^{n_{0}}\right) \circ \mu_{s}^{n}-\mathscr{H}_{m_{0}}^{t}\left(\dot{\mu}_{s}^{n_{0}}\right) \circ \mu_{s}^{n}\right| d s \\
& +\int_{0}^{1}\left|\mathcal{H}_{m_{0}}^{t}\left(\dot{\mu}_{s}^{n_{0}}\right) \circ \mu_{s}^{n}-\mathcal{H}_{m_{0}}^{t}\left(\dot{\mu}_{s}^{n_{0}}\right) \circ \mu_{s}^{m}\right| d s \\
& +\int_{0}^{1}\left|\mathcal{H}_{m_{0}}^{t}\left(\dot{\mu}_{s}^{n_{0}}\right) \circ \mu_{s}^{m}-\mathscr{H}_{m}^{t}\left(\dot{\mu}_{s}^{n_{0}}\right) \circ \mu_{s}^{m}\right| d s
\end{aligned}
$$

for some $m_{0}$.

Exactly like in the proof of sub-lemma 1

$$
G(t, n, m) \leq 2 E\left|\mathscr{H}_{m}^{t}-\mathscr{H}_{m_{0}}^{t}\right| .\left(l\left(\Psi^{n_{0}}\right)^{-1}\right)+F
$$

where

$$
F=\int_{0}^{1}\left|\mathcal{P}_{m_{0}}^{t}\left(\dot{\mu}_{s}^{n_{0}}\right) \circ \mu_{s}^{n}-\mathscr{X}_{m_{0}}^{t}\left(\dot{\mu}_{s}^{n_{0}}\right) \circ \mu_{s}^{m}\right| d s
$$

By uniform continuity of $\mathscr{H}_{m_{0}}^{t}\left(\dot{\mu}_{s}^{n_{0}}\right), F \rightarrow 0$ when $n, m \rightarrow \infty$ since $\mu_{t}^{n}$ is Cauchy.

By similar arguments as in the sub-lemma $1, G \rightarrow 0$ and hence $L \rightarrow 0$ when $m, n \rightarrow \infty$ and $m_{0} \rightarrow \infty$.

We have just proved that the subset $\operatorname{SSymp}(M, \omega)$ of $\operatorname{Symeo}(M, \omega)$ is closed under composition and inversion. This concludes the proof that $\operatorname{SSympeo}(M, \omega)$ is a group.

The fact that it is arcwise connected in the ambiant topology of $\operatorname{Homeo}(M)$ is obvious from the definition.

$\operatorname{Hameo}(M, \omega)$ is a normal subgroup of $\operatorname{SSympeo}(M, \omega)$ since it is normal in $\operatorname{Sympeo}(M, \omega)$ [13].

Let $h, g \in S \operatorname{Sympeo}(M, \omega)$ and let $\Phi^{n}, \Psi^{n}$ be symplectic isotopies which form Cauchy sequences and $C^{0}$ converge to $h, g$. By the main lemma the sequence $\Phi^{n} \cdot \Psi^{n} \cdot\left(\Phi^{n}\right)^{-1}\left(\Phi^{n}\right)^{-1}$ is a Cauchy sequence. It obviously converges $C^{0}$ to the commutator $h g h^{-1} g^{-1} \in \operatorname{SSympeo}(M, \omega)$.

It is a standard fact that $\Phi^{n} . \Psi^{n} .\left(\Phi^{n}\right)^{-1}\left(\Psi^{n}\right)^{-1}$ is a hamiltonian isotopy.

Indeed let $\phi_{t}$ and $\psi_{t}$ be symplectic isotopies, and let $\sigma_{t}=\phi_{t} \psi_{t} \phi_{t}^{-1} \psi_{t}^{-1}$, then

$$
\dot{\sigma}_{t}=X_{t}+Y_{t}+Z_{t}+U_{t}
$$


with $X_{t}=\dot{\phi}_{t}, Y_{t}=\left(\phi_{t}\right)_{*} \dot{\psi}_{t}, Z_{t}=-\left(\phi_{t} \psi_{t} \phi_{t}^{-1}\right)_{*} \dot{\phi}_{t}$, and $U_{t}=-\left(\sigma_{t}\right)_{*} \dot{\psi}_{t}$.

By proposition 5, $i\left(X_{t}+Z_{t}\right) \omega$ and $i\left(Y_{t}+U_{t}\right) \omega$ are exact 1-forms. Hence $\sigma_{t}$ is a hamiltonnian isotopy.

By Proposition 1, the metric $D$ coincides with the one for hamiltonian isotopies. Hence $\Phi^{n} \cdot \Psi^{n} \cdot\left(\Phi^{n}\right)^{-1}\left(\Psi^{n}\right)^{-1}$ is a Cauchy sequence for $d_{\text {ham }}$. Therefore: [SSympeo $(M, \omega)$, SSympeo $(M, \omega)] \subset \operatorname{Hameo}(M, \omega)]$. This concludes the proof of theorem 3

Proof of Theorem 2: We now prove that $\operatorname{SSympeo}(M, \omega)$, with the symplectic topology, is a topological group.

In fact, we prove that $\overline{\mathscr{Q}}$ ( see section 3 ) is a topological group. Recall that an element of $\overline{\mathscr{Q}}$ is a couple $(\gamma, V=(\not{H}, u)$

where $\gamma \in \mathscr{P}$ Homeo $(M), \mathscr{H} \in L^{(1, \infty)}\left([0,1], \operatorname{harm}^{1}(M, \omega), u \in L^{(0,1)}([0,1] x M, \mathbb{R})\right.$, and there exists a $d_{\text {symp }}$ - Cauchy sequence of symplectic isotopies $\Phi_{n}(t)$ such that $\Phi_{n}(1) \rightarrow \gamma$, in the $C^{0}$ topology and $\lim _{n \rightarrow \infty}\left(\mathscr{H}_{n}, u_{n}\right)=(\mathscr{H}, u)$. Here we wrote $\mathscr{H}_{n}$ for $\mathscr{H}^{\Phi_{n}}$ and $u_{n}$ for $u_{n}^{\Phi_{n}}$.

The product and the inverse in $\overline{\mathscr{Q}}$ are given by:

$$
\begin{gathered}
(\gamma,(\mathscr{H}, u)) \cdot\left(\gamma^{\prime},\left(\mathscr{H}^{\prime}, u^{\prime}\right)\right)=\left(\gamma \gamma^{\prime},\left(\mathscr{H}+\mathscr{H}^{\prime}, u+u^{\prime} \circ \gamma+v\right)\right) \\
(\gamma,(\mathscr{H}, u))^{-1}=\left(\gamma^{-1},(-\mathscr{H},-(u \circ \gamma+w))\right.
\end{gathered}
$$

where $v$ is the limit of the Cauchy sequence $v_{n}(t)$ given by formula (II):

$$
v_{n}(t)=\int_{0}^{1}\left(\mathscr{H}_{n}^{\prime}\left(\dot{\sigma}_{n}(s)\right) \circ \sigma_{n}(s)\right) d s,
$$

with $\sigma_{n}(s)=\left(\Phi_{n}^{\prime}(s)\right)^{-1}$. and $w$ the limit of a similar sequence in which $\sigma_{n}$ is replaced by $\Phi_{n}$.

Part I. Let us first show that the inversion is continuous: let $\left(\gamma_{k},\left(\mathscr{H}_{k}, u_{k}\right)\right)$ be a sequence converging to $(\gamma,(\mathscr{H}, u))$, For each $k$, there is a Cauchy sequence $\Phi_{n}^{k}$ of symplectic isotopies such that $\Phi_{n}^{k} \rightarrow \gamma_{k}$ as $n \rightarrow \infty$ in the $C^{0}$ topology, $\mathscr{H}_{n}^{k} \rightarrow \mathscr{H}_{k}, u_{n}^{k} \rightarrow u_{k}$.

We need only to show that $w_{k} \rightarrow w$, that is $(*)$

$$
\lim _{n, k \rightarrow \infty} \int_{0}^{1}\left|\mathscr{X}_{n}^{k}\left(\dot{\Phi}_{n}^{k}(s)\right) \circ \Phi_{n}^{k}(s)-\mathscr{H}_{n}\left(\dot{\Phi}_{n}(s)\right) \circ \Phi_{n}(s)\right| d s=0 .
$$

We have the following inequalities :

$$
\left\|\dot{\Phi}_{n}^{k}-\dot{\Phi}_{n}\right\| \leq\left\|\dot{\Phi}_{n}^{k}-V^{k}\right\|+\left\|V^{k}-V\right\|+\left\|V-\dot{\Phi}_{n}\right\|
$$

and each term in the right hand of this inequality $\rightarrow 0$ as $n, k \rightarrow \infty$.

Similarly,

$$
\left|\mathscr{H}_{n}^{k}-\mathscr{H}_{n}\right| \leq\left|\mathscr{H}_{n}^{k}-\mathscr{H}^{k}\right|+\left|\mathscr{H}^{k}-\not{H}\right|+\left|\not{H}-\mathscr{H}_{n}\right|
$$


and each term in the right hand of this inequality $\rightarrow 0$ as $n, k \rightarrow \infty$.

Formula $(*)$ follows from these inequalities and the techniques developped in this paper (including the reparametrisation lemma). We leave the details to the reader.

Part II. Now we prove that the composition is continuous: let $\left(\gamma^{k}, V^{k}=\left(\mathscr{C}^{k}, u^{k}\right)\right)$ and $\left(\gamma^{\prime k}, V^{\prime k}=\right.$ $\left.\left(\mathscr{H}^{\prime k}, u^{\prime k}\right)\right)$ converging to $(\gamma,(\mathscr{H}, u))$ and $\left(\gamma^{\prime},\left(\mathscr{C}^{\prime}, u^{\prime}\right)\right)$.

By part I, if $\dot{\sigma}_{n}^{k} \rightarrow U^{k}$ and ${\dot{\sigma^{\prime}}}_{n}^{k} \rightarrow U^{\prime k}$, then by part I, $U^{k} \rightarrow U$. Here we denoted by $\sigma_{n}^{k}$, and ${\sigma^{\prime}}_{n}^{k}$ respectively $\left(\Phi_{n}^{k}\right)^{-1},\left(\Phi_{n}^{\prime k}\right)^{-1}$.

We only need to prove:

1) $u^{k} \circ \gamma_{k} \rightarrow u \circ \gamma$

2) $v^{k} \rightarrow v$.

The proof of (1) goes along the lines explained in this paper ( including the reparamareization lemma ) and the details are left tothe reader.

The proof of (2) follows from part I and uses the inequalities:

$$
\left\|\dot{\sigma}_{n}^{k}-\dot{\rho}_{n}\right\| \leq\left\|\dot{\sigma}_{n}^{k}-U^{k}\right\|+\left\|U_{-}^{k} U\right\|+\left\|U-\dot{\rho}_{n}\right\|
$$

Each of the three parts of the second member of the inequality $\rightarrow 0$ as $n, k \rightarrow \infty$. The details are left tothe reader.

This concludes the proof of theorem 2.

Appendix: For the convenience of the reader, we give here the proofs of propositons 3, 4, and 5 .

Proof of Proposition 3: Let $\theta$ be a p-form, $X$ a vector field and $\phi$ a diffeomorphism. For any $x \in M$ and any vector fields $Y_{1}, . . Y_{p-1}$, we have:

$$
\begin{aligned}
& \left(\phi^{-1}\right)^{*}\left[i_{X} \phi^{*} \theta\right](x)\left(Y_{1}, \ldots, Y_{p-1}\right)=\left(i_{X} \phi^{*} \theta\right)\left(\phi^{-1}(x)\right)\left(D _ { x } \phi ^ { - 1 } \left(Y_{1}(x), \ldots\left(D_{x} \phi^{-1}\left(Y_{p-1}(x)\right)\right.\right.\right. \\
& =\left(\phi^{*} \theta\right)\left(\phi^{-1}(x)\right)\left(X_{\phi^{-1}(x)}, D_{x} \phi^{-1}\left(Y_{1}(x)\right), \ldots\left(D_{x} \phi^{-1}\left(Y_{p-1}(x)\right)\right.\right. \\
& =\theta\left(\phi ( \phi ^ { - 1 } ( x ) ) \left(D_{\phi^{-1}(x)} \phi\left(X_{\phi^{-1}(x)}\right), D_{\phi^{-1}(x)} \phi D_{x} \phi^{-1}\left(Y_{1}(x)\right), \ldots D_{\phi^{-1}(x)} \phi D_{x} \phi^{-1}\left(Y_{p-1}(x)\right.\right.\right. \\
& =\theta(x)\left(\left(\phi_{*} X\right)_{x}, Y_{1}(x), . . Y_{p-1}(x)\right) \\
& =\left(i\left(\phi_{*} X\right) \theta\right)(x)\left(Y_{1}, \ldots, Y_{p-1}\right) \\
& \text { since } D_{\phi^{-1}(x)} \phi D_{x} \phi^{-1}=D_{x}\left(\phi \phi^{-1}\right)=i d .
\end{aligned}
$$

Therefore $\left.\left(\phi^{-1}\right)^{*}\left[i_{X} \phi^{*} \theta\right]=i\left(\phi_{*} X\right)\right) \theta$

Proof of Proposition 4: This is just the chain rule. See [10] page 145. 
Proof of proposition 5: For a fixed $t$, we have

$$
\frac{d}{d s} \phi_{s}^{*} \theta_{t}=\phi_{s}^{*}\left(L_{\dot{\phi}_{s}} \theta_{t}\right),
$$

where $L_{X}$ is the Lie derivative in the direction $X$. Since $\theta$ is closed, we have:

$$
\frac{d}{d s} \phi_{s}^{*} \theta_{t}=\phi_{s}^{*}\left(d i_{\dot{\phi}_{s}} \theta_{t}\right)=d\left(\phi_{s}^{*}\left(\theta_{t}\left(\dot{\phi}_{s}\right)\right)=d\left(\theta_{t}\left(\dot{\phi}_{s}\right) \circ \phi_{s}\right) .\right.
$$

Hence for every $u$

$$
\phi_{u}^{*} \theta_{t}-\theta_{t}=\int_{0}^{u} \frac{d}{d s} \phi_{s}^{*} \theta_{t} d s=d\left(\int_{0}^{u}\left(\theta_{t}\left(\dot{\phi}_{s}\right) \circ \phi_{s}\right) d s\right)
$$

Now set $u=t$.

\section{Acknowledgement}

I would like to thank Claudio Cuevas for soliciting this paper for Cubo.

I am also very grateful to the referee for an extensive list of good remarks, questions, and suggestions which drastically improved the final form of this paper. In particular I owe to him/her the idea to finish the Proof of Theorem 1.

\section{References}

[1] Banyaga, A., On the group of symplectic homeomorphisms, C.R. Acad. Sci. Paris Ser., 1 346(2008), 867-872.

[2] Banyaga, A., A Hofer-like metric on the group of symplectic diffeomorphisms, Contemp. Math., Vol. 512 (2010), 1-23.

[3] Banyaga, A., Sur la structure du groupe des difféomorphismes qui préservent une forme symplectique, Comment. Math. Helv., 53(1978), pp.174-227.

[4] Banyaga, A., The structure of classical diffeomorphisms groups, Mathematics and its applications vol 400. Kluwer Academic Publisher's Group, Dordrecht, The Netherlands, 1997.

[5] Banyaga, A. And Spaeth, P., The group of contact homeomorphisms, Preprint, 2008.

[6] Banyaga, A. And Spaeth, P., The $C^{0}$ contact topology and the group of contact homeomorphisms, ArXiv 0812.2461.

[7] Eliashberg, Y.M., A theorem on the structure of wave fronts and its application in symplectic topology, Funct. Anal. and Its Applications, (1987), 21:227-232. 
[8] FAthi, A., Structure of the group of homeomorphisms preserving a good measure on a compact manifold, Ann. Scient. Ec. Norm. Sup., 13(1980), 45-93.

[9] Gromov, M., Partial differential relations, Ergebnisse der Mathematik, Springer, 1986.

[10] Hofer, H. And Zehnder, E., Symplectic invariants and hamiltonian dynamics, Birkhauser Advanced Texts, Birkhauser Verlag, 1994.

[11] Lalonde, F., McDuff, D. And Polterovich, L., Topological rigidity of hamiltonian loops and quantum homology, Invent. math., 135(1999), 369-385.

[12] MüLlER, S., The group of hamiltonian homeomorphisms in the $L^{\infty}$ norm, J. Korean Math.Soc., 45(2008) no6., 1769-1784.

[13] OH, Y-G. AND MüLLER, S, The group of hamiltonian homeomorphisms and $C^{0}$ symplectic topology, J. Symp. Geometry, 5(2007), 167-225.

[14] WARner, F., Foundations of differentiable manifolds and Lie groups, Scott, Foresman and Company, 1971. 